\title{
A model for the interpretation of nuclear magnetic resonance spin-lattice dispersion measurements on mortar, plaster paste, synthetic clay and oil-bearing shale
}

\author{
D. A. Faux and P. J. McDonald \\ Department of Physics, University of Surrey, Guildford, Surrey GU2 7XH, United \\ Kingdom
}

\begin{abstract}
A model linking the molecular-scale dynamics of fluids confined to nanopores to nuclear magnetic resonance (NMR) relaxation rates is proposed. The model is used to re-analyse fast field-cycling spin-lattice relaxation rate measurements for the separate water and oil dispersions from an oil-bearing shale [Korb et al, J. Phys. Chem. C, 118, 199 (2014)]. The model assumes that pore fluid can be characterized by three time constants: the surface and bulk diffusion correlation times and a surface desorption time constant. Results are shown to yield meaningful and consistent intra-pore dynamical time constants, insight into diffusion mechanisms and pore morphology. The shale is found to be oil-wetting and the water dispersion is found to be due to the interaction of aqueous $\mathrm{Mn}^{2+}$ ions with bulk water spins. Clay, mortar and plaster paste dispersions measurements have also been successfully reanalysed and a summary of the results is presented. The results demonstrate the wide applicability of the model which advances NMR dispersion experimentation as a powerful tool for measuring nano-porous fluid properties
\end{abstract}

Keywords: NMR dispersion, fast-field cycling, porous media, oil-bearing shale

Fast-field-cycling NMR dispersion (NMRD) relaxometry has been applied, for decades, to a broad range of systems including cement-based materials, bio-polymers, glasses, plaster, rock and more $[1,2,3,4,5]$. The spin-lattice (longitudinal) relaxation rate $T_{1}^{-1}$ is measured over frequencies 
typically in the range $0.01-40 \mathrm{MHz}$ and therefore captures information on the relative motion of spins over many orders of magnitude in time. However, for NMRD to be useful, a model is required to link fluid molecular dynamics in pores to the frequency-dependence of $T_{1}^{-1}$. To achieve this, spin dynamics must be described by a diffusion model from which the dipolar correlation function, $G(t)$, is calculated. $G(t)$ is a measure of the time-dependence of the dipolar interaction of pairs of spins which are moving relative to each other and the theoretical challenge lies in the determination of $G(t)$ for the model of choice. Once $G(t)$ has been calculated, its Fourier transform yields the spectral density function $J(\omega)$ which is simply related to both $T_{1}^{-1}$ and the spin-spin (transverse) relaxation rate $T_{2}^{-1}$. The model dispersion can then be fit to the experimental $T_{1}^{-1}$ dispersion curve and combined with separate single-frequency measurements of the ratio $T_{1} / T_{2}$ if available.

A family of models which has been widely used and has proved successful at fitting $T_{1}^{-1}$ dispersions across a broad range of systems is due to Korb and co-workers $[2,3,4,5]$. We refer to the collection of models as the "Korb model". One frequently-applied Korb model identifies the key contribution to the relaxation rate as that due to the interaction of mobile surface spins with a layer of paramagnetic impurities assumed to lie within the same twodimensional (2D) plane as the mobile ${ }^{1} \mathrm{H}$ spins at the pore surface. One nano-scale diffusion correlation time emerges from a fit to an experimental dispersion curve using the Korb model: the surface layer diffusion correlation time $\tau_{\ell} . \tau_{\ell}$ is arbitrarily defined as the time for a spin to move $\delta=0.27 \mathrm{~nm}$ in $3 \mathrm{D}$ so that the diffusion coefficient $D=\delta^{2} / 6 \tau_{\ell}$. A second time parameter, the desorption time constant $\tau_{d}$, may be obtained from a separate measurement of the $T_{1} / T_{2}$ ratio at a single frequency. $\tau_{d}$ is the time after which the number of mobile spins on the surface falls to $e^{-1}$ of its value at $t=0$ due to desorption.

A difficulty with the interpretation of the time constants that emerge from the application of a Korb model is that, regardless of the system, fits to experimental dispersion curves find $\tau_{\ell}$ to be shorter than $\tau_{d}$ by typically $2-5$ orders of magnitude. This implies 100-100000 hops of a spin bound to the surface before desorption. The issue is most apparent in the oil-bearing shale system [5] where $\tau_{\ell} \approx 10 \mathrm{ps}$ is found for the surface water. Pure bulk water at room temperature has a correlation time of 5 ps implying that water can diffuse nearly at the same rate as pure bulk water whilst being bound to a surface and make about 100000 hops on the surface prior to desorption.

In this article we propose an alternative model which we refer to as the $3 \tau$ model. The model is illustrated schematically in Fig. 1. The $3 \tau$ model 
incorporates the key physics of the Korb model but contains $\tau_{\ell}, \tau_{d}$ and $\tau_{b}$ as explicit time constants ${ }^{1}$ where $\tau_{b}$ is the bulk fluid diffusion correlation time constant. The $3 \tau$ model approximates the complex geometry of a pore as a quasi-two-dimensional (Q2D) system with locally-flat surfaces. The surface layer is assumed to comprise a monolayer with the remaining fluid adopting bulk-like properties, broadly consistent with molecular dynamics simulation $[6,7]$.

In order to translate the $3 \tau$ model to predictions of $T_{1}^{-1}$, expressions must be derived for the various interactions between pairs of spins. One important innovation in the $3 \tau$ model, as applied to systems in which $T_{1}^{-1}$ is dominated by interactions with electronic paramagnetic impurity spins, is the placement of the effective layer of paramagnetic spins below the pore surface as opposed to within the surface mobile layer as a pure 2D layer as in the Korb model. By placing the effective paramagnetic layer below the surface, the long-time limit of $G(t)$ decays as $t^{-2}$ (rather than $t^{-1}$ when all spin species are contained in a pure $2 \mathrm{D}$ layer) leading to fundamentally different $T_{1}^{-1}$ dispersions and a different value of the time constant $\tau_{\ell}$ when the model is fit to experimental data. The $t^{-2}$ dependence of $G(t)$ has been previously demonstrated theoretically and computationally $[6,8,9]$. A second innovation is that we integrate across the full thickness of the fluid to correctly account for the strong distance-dependence of the dipolar interaction. This process enables a direct estimate of $\tau_{b}$ (and hence the diffusion coefficient of the bulk fluid) from the high-frequency limit of the dispersion curve. The model has limitations, of course. For example, it does not explicitly include molecular spin rotation. Indeed, we are not aware of any model that properly accounts for rotation as well as translation of molecules in confined geometries where the rotational correlation time may be site dependent. A full and frank analysis of the limitations of the $3 \tau$ model is presented in Ref. [9].

In this article, we provide a detailed re-analysis of the water and oil $T_{1}^{-1}$ dispersions for an oil-bearing shale. The experimental results were obtained by Korb and co-workers [5] and to our knowledge constitute the only study of its kind on an oil-bearing shale. We also summarise the results of the application of the $3 \tau$ model to three further systems of interest to different research communities: a plaster paste, a synthetic saponite clay and a mortar.

\footnotetext{
${ }^{1}$ This is effectively the same number of time constants as the Korb model since the Korb model additionally invokes the bulk fluid relaxation time without frequency dependence.
} 
These systems additionally test the model under conditions of different paramagnetic doping and hence surface relaxivity. The summary data provides confidence in the validity of the $3 \tau$ model and reveals a wealth of information into the fluid dynamics, diffusion mechanisms and pore morphology which has hitherto been inaccessible.

\section{Results and discussion}

The expressions required to describe all the combinations of spin-pair interactions which contribute to the relaxation dispersion for a specific system have been presented $[8,9]$. The $3 \tau$ model is applied to four systems: an oilbearing shale (water and oil), a plaster paste, mortar and saponite clay. In each case, the fit quality is assessed by a least-squares goodness-of-fit parameter $Q$ which is minimised for the $\tau_{\ell}, \tau_{d}, \tau_{b}$ space. There is only a single minimum found for each system. The uncertainties in the time constants presented in the tables below represent a $3 \%$ variation from the optimum $Q$ except where the experimental $T_{1} / T_{2}$ ratio is known in which case the range of $\tau_{\ell}\left(T_{1} / T_{2}\right.$ is primarily sensitive to $\tau_{\ell}$ here) reflects the experimental range of $T_{1} / T_{2}$. We emphasise that, in all cases where $T_{1} / T_{2}$ is known, the range of optimum $\tau_{\ell}\left(Q_{\min }+3 \%\right)$ coincides with the experimental range of $T_{1} / T_{2}$. This fact provides further confidence in the validity of the model.

\subsection{Application of the $3 \tau$ model to an oil-bearing shale}

Here we apply the $3 \tau$ model to an oil-bearing shale: a complex two-fluid system of topical interest. The experimental oil and water dispersions are presented in Fig. 2 and are taken from Korb and co-workers [5]. This is the only NMRD measurement on an oil-bearing shale performed to date. The authors explain the process by which the separate oil and water dispersion curves are obtained and readers are referred to Ref. [5] for the experimental details.

Spin relaxation is due to the interaction of ${ }^{1} \mathrm{H}$ in the water and oil with $\mathrm{Mn}^{2+}$ ions (labelled " $\ell$ ") which were identified as the dominant paramagnetic species by electron spin resonance [5]. However, satisfactory fits cannot be obtained using the $3 \tau$ model with the experimental $\mathrm{Mn}^{2+}$ spin density of $N_{\sigma} \approx 0.5 / \mathrm{nm}^{3}[5]$. It is found that the effective $\mathrm{Mn}^{2+}$ density is about $N_{\sigma} / 20$. We therefore use $N_{\sigma}$ as an additional fit parameter. Furthermore, although the pore surface is found to be oil wetting (a conclusion supported by the high values of the relaxation rate), the shape of the dispersion was found to 
be inconsistent with an even oil coverage at the rock surfaces. The model was therefore adapted to allow a fraction $f$ of the pore surface to be covered by a monolayer of oil with water above leaving a fraction $(1-f)$ of the surface covered by a thicker layer of oil. Where the surface is covered by an oil monolayer, desorption does not take place because desorption would move an oil molecule into water. The surface fraction containing a thicker layer of oil allows for surface oil to desorb into the bulk and therefore allows surface and bulk oil to exchange.

Consequently, the contribution $G_{\sigma \ell}^{\text {oil }}(t)$ may be written

$$
G_{\sigma \ell}^{\mathrm{oil}}(t)=f G_{\sigma \ell}(t)+(1-f) G_{\sigma \ell}(t) e^{-t / \tau_{d}}
$$

where $G_{\sigma \ell}(t)$ is calculated using Eqs. (17)-(20) from Ref. [9] using parameters in Table 1 for surface oil. The exponential is introduced to account for the desorption of surface oil into bulk oil. Several pore models could lead to Eq. (1) such as that illustrated in Fig. 3 which shows a fraction $(1-f)$ of the oil in surface pits, or droplets of oil at the surface. Trial fitting found that the best fits arise for $f \approx 0.2$ and so $f$ was fixed to this value.

Table 1: List of model parameters required to fit to the $T_{1}^{-1}$ dispersion [5] for oil and water in oil-bearing shale. $d_{\sigma \ell}\left(d_{\sigma b}\right)$ are distances between an effective layer of paramagnetic impurities in the crystal and the front face of the surface layer (bulk) fluid. $\delta=0.27 \mathrm{~nm}$ and $h$ is the thickness of the fluid layer. The values for the $\tau$ time constants emerging from the fits as described in the text are listed.

\begin{tabular}{lll} 
Parameter & Oil & Water \\
\hline$f$ & 0.2 & - \\
$d_{\sigma \ell} / d_{\sigma b}$ & $2 \delta / 3 \delta$ & $-/ 3 \delta$ \\
$h_{\ell} / h_{b}$ & $\delta / 18 \delta$ & $-/ 18 \delta$ \\
$\tau_{b}$ & $20-40 \mathrm{ps}$ & $10-40 \mathrm{ps}$ \\
$\tau_{\ell}$ & $0.1-0.5 \mu \mathrm{s}$ & - \\
$\tau_{d}$ & $0.2-0.3 \mu \mathrm{s}$ & - \\
\hline \hline
\end{tabular}

$G_{\sigma b}(t)$ is calculated using Eqs. (17)-(20) from Ref. [9] using parameters in Table 1. The final parameters and fit outcomes are listed in Table 1. The bulk oil correlation time $\tau_{b}$ lies in the range $20-40 \mathrm{ps}$, consistent with, but slightly longer than, typical pure alkanes (6-11 ps [10]). The $T_{1, \text { oil }} / T_{2, \text { oil }}$ ratio, which ranges from 5 to 10 experimentally [5], is found to be a strong function of the surface diffusion correlation time $\tau_{\ell}$ with $\tau_{\ell}=0.1 \mu$ s corresponding to 
$T_{1, \text { oil }} / T_{2, \text { oil }} \approx 5$ and $\tau_{\ell}=0.5 \mu$ s to $T_{1, \text { oil }} / T_{2, \text { oil }} \approx 10$. This result suggests that the $T_{1, \text { oil }} / T_{2, \text { oil }}$ ratio might provide a direct measure of surface affinity. It may be possible to infer oil chain length and surface affinity from $T_{1}-T_{2}$ maps when combined with peak-spread information. With down-bore $T_{1}-T_{2}$ mapping a possibility in the future, the significance of this result is obvious.

The water component of the experimental dispersion, $T_{1 \text {,wat }}^{-1}$, is presented in Fig. 2. The magnitude of $T_{1, \text { wat }}^{-1}$ for the experimental water dispersion data provides unequivocal evidence of interaction with $\mathrm{Mn}^{2+}$ ions but the numerical values of the experimental relaxation rate are less than those from the oil. Indeed, if we attempt to fit to the water dispersion using the same model as for the oil (assuming surface and bulk water) we find that fits are incompatible with the presence of surface water. Simply, for any choice of $\tau_{\ell}, \tau_{d}$ and $\tau_{b}$, it is found that the optimum fit is obtained if the surface water contribution is zero. The shallow decay of the water dispersion curve as a function of frequency indicates that the water is fast-moving and therefore not located on the pore surface within the $3 \tau$ model - an independent observation compatible with an oil-wetting shale.

It is therefore proposed that the water dispersion contains a contribution due to aqueous $\mathrm{Mn}^{2+}$ ions in the bulk water. This conclusion is supported by the earlier observation that $\mathrm{Mn}^{2+}$ impurities are depleted at the pore surfaces, presumably having desorbed over millennia into the bulk. It is noted that $\mathrm{Mn}^{2+}$ was not found in the oil where it is insoluble. The contribution $T_{1, \text { aq }}^{-1}$ due to aqueous $\mathrm{Mn}^{2+}$ is estimated from the expression obtained for bulk water $[11,12]$ adapted to describe the relative motion of water spins with respect to a $\mathrm{Mn}^{2+}$ ion assumed to be static. Therefore

$$
T_{1, \text { wat }}^{-1}\left(\tau_{b}\right)=T_{1, \sigma b}^{-1}\left(\tau_{b}\right)+T_{1, \text { aq }}^{-1}\left(\tau_{b}\right)
$$

which has a single fit time constant, $\tau_{b}$. $T_{1, \sigma b}^{-1}$ is calculated using Eqs. (17)-(20) from Ref. [9] with the parameters listed in Table 1 for water. Optimum fits are obtained for $\tau_{b} \approx 10-40 \mathrm{ps}$ with the wide range reflecting the uncertainty in determining the asymptotic limit of the dispersion at the higher frequencies. The aqueous $\mathrm{Mn}^{2+}$ density is found to lie in the range 5-7.5 mM for both the oil and water dispersions, a factor 100-150 more dilute than the measured equivalent density in the solid.

Korb and co-workers [5], in their original analysis of the shale system, concluded that the shale was water wetting rather than oil-wetting as found here. We therefore undertook two additional calculations assuming a waterwetting shale in which the shale pores were assumed to contain a monolayer 
of water only with no bulk water. The sole time constant for the fitting is therefore $\tau_{\ell}$. If the spins in the surface water monolayer move with $\tau_{\ell}=1 \mu \mathrm{s}$ (typical of other rock-like systems), the fit is poor as illustrated in Fig. 2. If $\tau_{\ell}=10 \mathrm{ps}$, as concluded by Korb in Ref. [5], the fit is much improved. Both the analysis in Ref. [5] and our calculations presented here lead to the conclusion that the water dispersion curve is associated with fast-moving water but the conclusions as to the water environment responsible for the dispersion differ. Our conclusion is that the water dispersion is associated with the rapid motion of water spins close to aqueous $\mathrm{Mn}^{2+}$ ions whereas Korb [5] concludes that the water moves rapidly close to the rock surface.

\subsection{Application of the $3 \tau$ model to plaster, clay and mortar}

We have applied the $3 \tau$ model to a further three systems of broad interest to the porous media community: a plaster paste with the experimental dispersion presented in Ref. [4], a synthetic saponite clay composed of stacked clay layers [1] and a mortar [3]. The plaster and clay systems test the $3 \tau$ model for systems where paramagnetic impurities are absent and so the relaxation rate is associated with the interactions between spins in the surface monolayer and the bulk. The underlying theory, the fitting process and detailed analyses have been presented $[8,9]$. A summary of the results is presented in Table 2 together with the outcomes of the original analysis.

Table 2: A summary of the fluid dynamical time constants that arise from fits to experimental $T_{1}^{-1}$ dispersions.

\begin{tabular}{lllll}
\hline \hline System & Model & $\tau_{\ell}$ & $\tau_{d}$ & $\tau_{b}$ \\
\hline \multirow{2}{*}{ Plaster paste [4] } & $\begin{array}{l}\text { Korb/Levitz [4, 13, 14] } \\
3 \tau\end{array}$ & $\begin{array}{l}5 \mathrm{~ns} \\
40 \pm 5 \mu \mathrm{s}\end{array}$ & $6.5 \pm 0.5 \mu \mathrm{s}$ & $17.0 \pm 0.5 \mathrm{ps}$ \\
\hline \multirow{2}{*}{ Saponite clay [1] } & BMSD & $1.8 \mu \mathrm{s}$ & & \\
& $3 \tau$ & $1.5 \pm 0.3 \mu \mathrm{s}$ & $5.0 \pm 0.5 \mu \mathrm{s}$ & $15 \pm 3 \mathrm{ps}$ \\
\hline \multirow{2}{*}{ Mortar [3, 15] } & Korb [3, 15] & $1.3 \mathrm{~ns}$ & $13 \mu \mathrm{s}$ & \\
& $3 \tau$ & $0.2-1.0 \mu \mathrm{s}$ & $0.8-1.1 \mu \mathrm{s}$ & $36 \pm 4 \mathrm{ps}$ \\
\hline \hline
\end{tabular}

We find that $\tau_{b}$ is approximately 3 times longer than the value for pure water at room temperature (5.3 ps [16]) for both the plaster and the synthetic 
clay with small uncertainties. The $3 \tau$ model finds $\tau_{\ell} \approx \tau_{d}$ in all three systems consistent with molecular dynamics simulations $[6,7]$. This result suggests, unsurprisingly, that the surface water diffusion rate is linked to the rate of desorption. In other words, a move of a surface molecule to a neighboring site may be linked to the departure of a molecule into the bulk leaving behind a vacancy site.

The saponite clay sample was originally analysed using the bulk-mediated surface diffusion (BMSD) model incorporating Lévy dynamics [1]. Here, $\tau_{\ell}$ for the synthetic clay from the $3 \tau$ and BMSD models are in excellent agreement. It is noted that the inclusion of the desorption constant $\tau_{d}$ into the model is sufficient to flatten the dispersion curve at low frequencies and to secure a high-quality fit. In the original work, a fit to the low-frequency plateau required the inclusion of Lévy dynamics $[1,17]$.

The re-analysis of the mortar sample [3] produced the time constants presented in Table 2. The dominant interactions here are between the paramagnetic impurities in the crystal and both the surface layer and bulk water. Both $\tau_{\ell}$ and $\tau_{d}$ are smaller for the mortar than the values obtained for the plaster and clay samples which suggests that the water is less strongly bound to the pore surfaces. Finally, for the mortar, $\tau_{b}$ is found to be 6 times the value of pure water at room temperature.

\section{Discussion and Conclusions}

A general model, referred to as the $3 \tau$ model, is used to characterize the molecular dynamics of fluids in porous solids using three time constants, the surface and bulk diffusion correlation times $\tau_{\ell}$ and $\tau_{b}$ respectively, and a desorption constant $\tau_{d}$. The model is tested by fitting to NMRD measurements of the separate water and oil $T_{1}^{-1}$ dispersion curves from an oil-bearing shale [5]. The analysis yields a wealth of physically-reasonable time constants which are consistent between the two co-existing fluids, provides insight into diffusion mechanisms and pore morphology. The oil-bearing shale is found to be oil-wetting and we find that the ratio $T_{1} / T_{2}$ is a strong function of $\tau_{\ell}$. The primary contributor to the water dispersion is believed to be associated with the movement of bulk water in the proximity of aqueous $\mathrm{Mn}^{2+}$ ions.

We have shown that the $3 \tau$ model may be applied to porous systems containing ${ }^{1} \mathrm{H}$ spins in motion relative to fixed paramagnetic impurities, such as mortar or oil shale, or to porous systems in which paramagnetic impurities 
are absent, such as plaster paste and synthetic saponite clay. Summary results are presented in Table 2 .

The $3 \tau$ model has been designed for porous systems in which the $T_{1}^{-1}$ dispersion is dominated by fluid contained in planar pores $h$. However, we have shown [9] that the $T_{1}^{-1}$ dispersion is independent of the pore thickness if the thickness is $5 \mathrm{~nm}$ or larger. Consequently, the $3 \tau$ model is also applicable to systems in which fluid is confined to channels or closed pores provided two of the three dimensions exceed $5 \mathrm{~nm}$. Furthermore, the similarity of $\tau_{\ell}$ and $\tau_{d}$ for all systems studied suggests that molecules execute typically 0-2 hops on the surface before desorption and therefore that the $3 \tau$ model is robust for systems presenting surfaces which are flat on the scale of $1 \mathrm{~nm}$. In principle, the model could be adapted to systems in which spin desorption is to a second layer that confines spins, such as molecular gels, protein or polymeric structures.

In summary, we believe that the $3 \tau$ model develops the NMRD experimental technique as a powerful tool for measuring the nanoscale dynamical properties of fluids in a wide range of porous solids.

This work was initiated under the Engineering and Physical Sciences Research Council (UK) (Grant number EP/H033343/1). We would also like to thank Jean-Pierre Korb for helpful discussions.

[1] T. Zavada, R. Kimmich, J. Grandjean, A. Kobelkov, J. Chem. Phys. 110 (1999) 6977.

[2] S. Godefroy, J.-P. Korb, M. Fleury, R. G. Bryant, Phys. Rev. E 64 (2001) 021605.

[3] F. Barberon, J.-P. Korb, D. Petit, V. Morin, E. Bermejo, Phys. Rev. Lett. 90 (2003) 116103.

[4] J.-P. Korb, New J. Phys. 13 (2011) 035016.

[5] J.-P. Korb, B. Nicot, A. Louis-Joseph, S. Bubici, G. Ferrante, J. Phys. Chem. C 118 (2014) 23212.

[6] D. A. Faux, P. J. McDonald, N. C. Howlett, J. S. Bhatt, S. V. Churakov, Phys. Rev. E 91 (2015) 032311.

[7] A. Striolo, MRS Bulletin 39 (2014) 1062-1067. 
[8] D. A. Faux, P. J. McDonald, N. C. Howlett, Phys. Rev. E 95 (2017) 033116 .

[9] D. A. Faux, P. J. McDonald, Phys. Rev. E 95 (2017) 033117.

[10] P. Blanco, B.-A. M. Mounir, J. K. Platten, P. Urteaga, J. A. Madariaga, C. Santamaria, J. Chem. Phys. 129 (17) (2008) 174504.

[11] A. Abragam, The Principles of Nuclear Magnetism, Oxford University Press, 1961.

[12] D. A. Faux, D. K. Ross, C. A. Sholl, J. Phys. C: Solid State Phys. 19 (1986) 4115.

[13] P. Levitz, J. Phys. Condens. Matter 17 (2005) S4059.

[14] P. Levitz, J.-P.Korb, Europhys. Lett.) 70 (2005) 684.

[15] P. J. McDonald, J.-P. Korb, J. Mitchell, L. Monteilhet, Phys. Rev. E 72 (2005) 011409.

[16] M. Holz, S. R. Heil, A. Sacco, Phys. Chem. Chem. Phys. 2 (2000) 4740.

[17] R. Kimmich, Chem. Phys. 284 (2002) 253. 


\section{FIGIJRES}
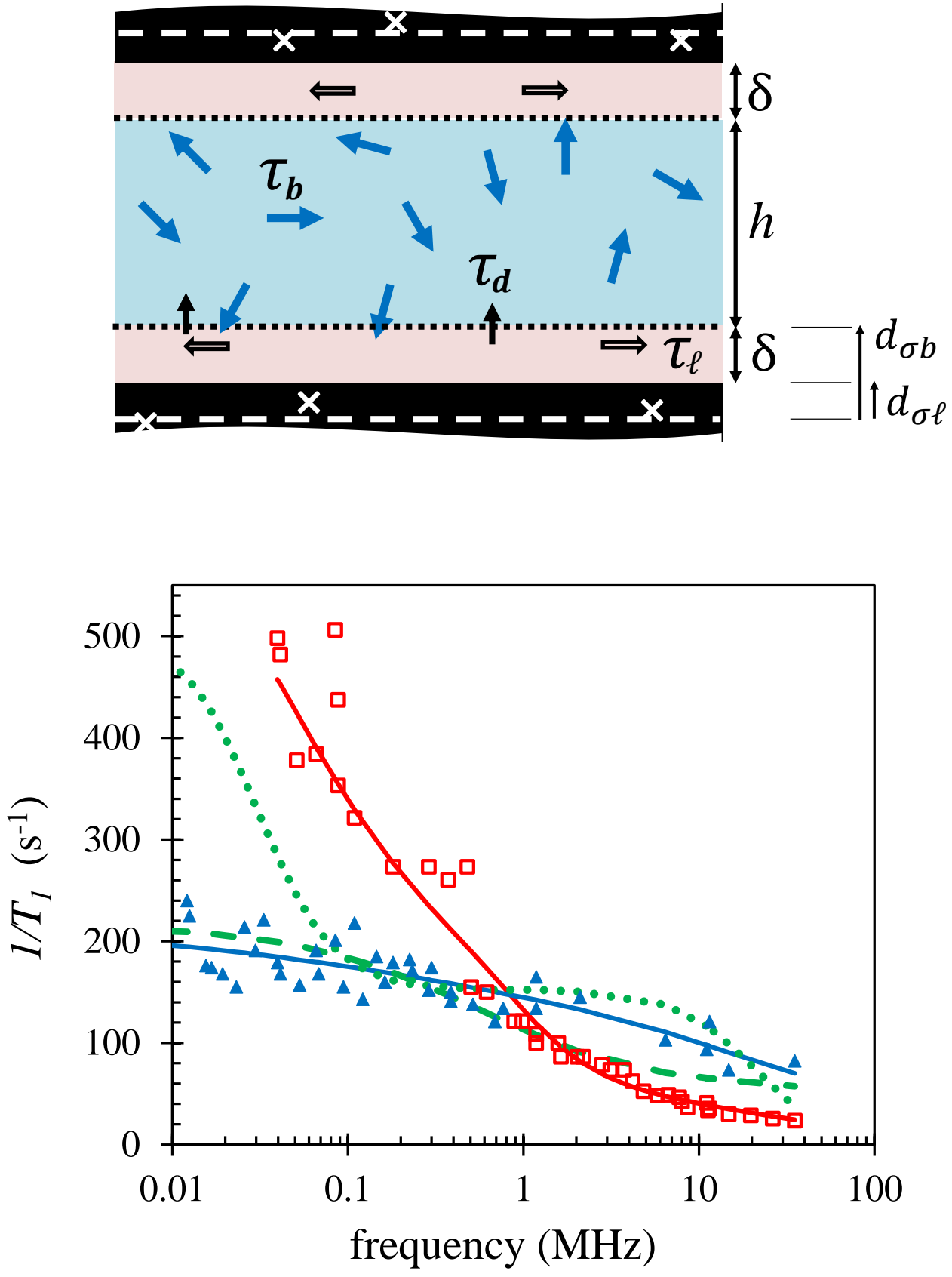


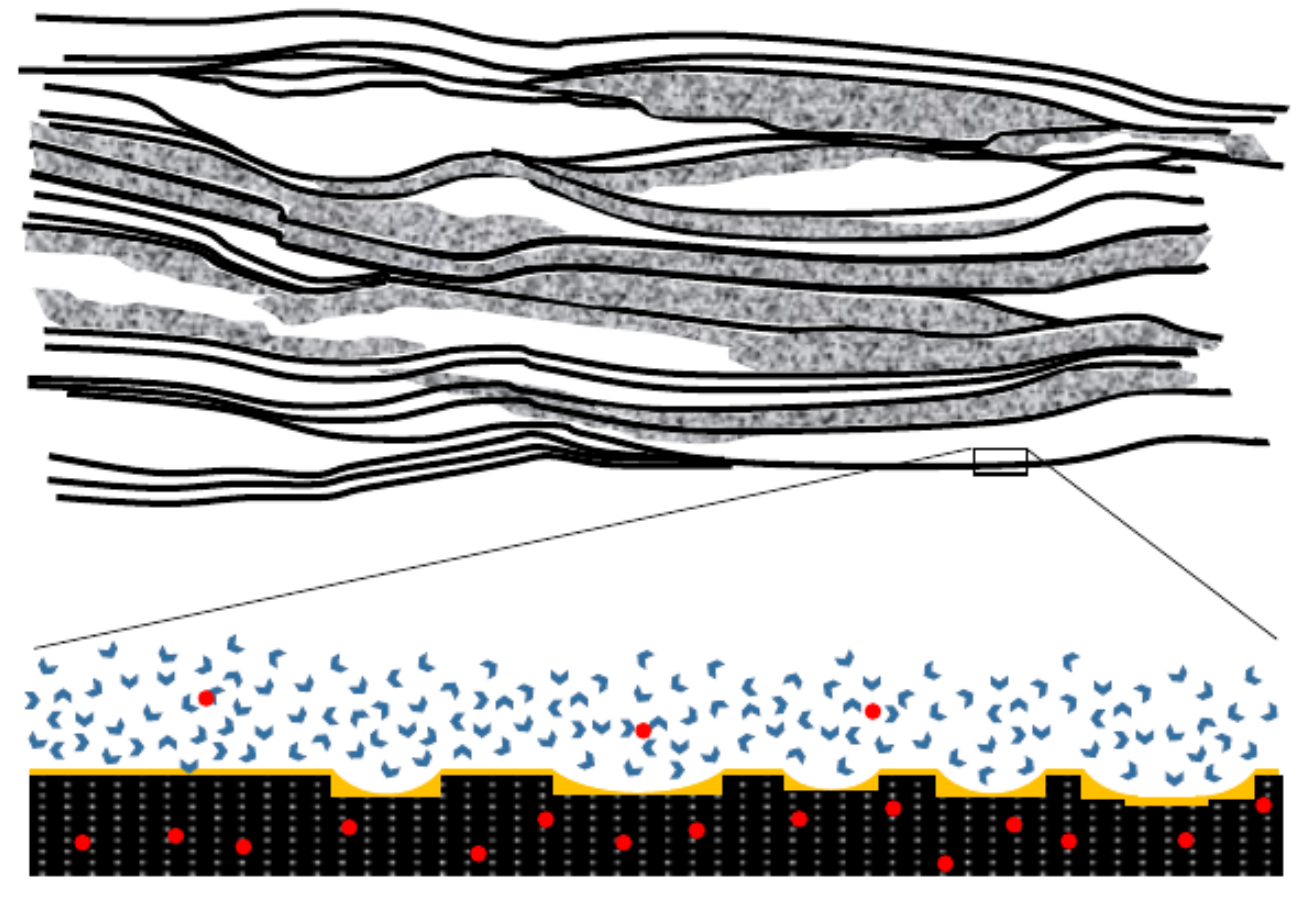




\section{CAPTIONS}

\section{Figure 1}

The $3 \tau$ model simplifies the complex motions of fluid confined to a planar (slab) pore to three diffusion time constants, $\tau_{\ell}, \tau_{d}$ and $\tau_{b}$, which characterize surface diffusion, desorption and bulk diffusion events respectively. The pore walls are black and contain rare paramagnetic impurities indicated by white crosses but modelled as a layer of uniform density (white dashed line). The pore contains bulk fluid of thickness $h$ (blue shading) and a surface monolayer of thickness $\delta=0.27 \mathrm{~nm}$ (pink shading). Distances from the paramagnetic layer to the front faces of the two fluid layers are labelled.

\section{Figure 2}

The experimental values for $T_{1, \text { oil }}^{-1}$ (squares) and $T_{1, \text { wat }}^{-1}$ (triangles) are presented as a function of frequency for an oil-bearing shale. The measurements were undertaken by Lorb and co-workers [5]. The solid lines are fits using the $3 \tau$ model described in the text for the oil and water components. Two additional curves are provided assuming a water-wetting shale containing a single surface monolayer of water (no oil): $\tau_{\ell}=1 \mu \mathrm{s}$ (green dotted line) and $\tau_{\ell}=10$ ps (green dashed line).

\section{Figure 3}

A schematic cartoon of an oil-bearing shale showing kerogen (gray texture) with an enlarged section illustrating a fluid model consistent with the analysis of the $T_{1}^{-1}$ dispersion measurements from Ref. [4]. Rock (dark shading), water (chevrons), oil $(\circlearrowleft)$ and $\mathrm{Mn}^{2+}$ paramagnetic impurities $(\bullet)$ are indicated. 